\title{
BASIC SCIENCE ARTICLE Dobutamine treatment reduces inflammation in the preterm fetal sheep brain exposed to acute hypoxia
}

\author{
Nadine Brew ${ }^{1}$, Shinji Nakamura ${ }^{1,2}$, Nadia Hale ${ }^{1}$, Aminath Azhan ${ }^{1}$, Grace I. Davies ${ }^{1}$, Ilias Nitsos ${ }^{1}$, Suzanne L. Miller ${ }^{1,3}$, \\ David W. Walker ${ }^{1,3,4}$ and Flora Y. Wong ${ }^{1,5,6}$
}

BACKGROUND: Impaired cerebral autoregulation in preterm infants makes circulatory management important to avoid cerebral hypoxic-ischemic injury. Dobutamine is frequently used as inotropic treatment in preterm neonates, but its effects on the brain exposed to cerebral hypoxia are unknown. We hypothesized that dobutamine would protect the immature brain from cerebral hypoxic injury.

METHODS: In preterm (0.6 gestation) fetal sheep, dobutamine (Dob, $10 \mu \mathrm{g} / \mathrm{kg} / \mathrm{min}$ ) or saline (Sal) was infused intravenously for $74 \mathrm{~h}$. Two hours after the beginning of the infusion, umbilical cord occlusion (UCO) was performed to produce fetal asphyxia (Sal +UCO: $n=9$, Dob+UCO: $n=7$ ), or sham occlusion (Sal+sham: $n=7$, Dob+sham: $n=6$ ) was performed. Brains were collected $72 \mathrm{~h}$ later for neuropathology.

RESULTS: Dobutamine did not induce cerebral changes in the sham UCO group. UCO increased apoptosis and microglia density in white matter, hippocampus, and caudate nucleus, and astrocyte density in the caudate nucleus. Dobutamine commenced before UCO reduced microglia infiltration in the white matter, and microglial and astrocyte density in the caudate.

CONCLUSION: In preterm hypoxia-induced brain injury, dobutamine decreases neuroinflammation in the white matter and caudate, and reduces astrogliosis in the caudate. Early administration of dobutamine in preterm infants for cardiovascular stabilization appears safe and may be neuroprotective against unforeseeable cerebral hypoxic injury.

Pediatric Research (2018) 84:442-450; https://doi.org/10.1038/s41390-018-0045-5

\section{INTRODUCTION}

Very preterm infants have an increased incidence of brain injury with accompanying motor, cognitive, memory, learning, and socialization deficits evident during childhood and beyond. ${ }^{1}$ Hypoperfusion of the neonatal brain can lead to cerebral hypoxia-ischemia and cerebral injury, particularly in the white matter. ${ }^{2}$ Effective circulatory management of extremely preterm infants with the aim of preventing cerebral hypoperfusion is therefore important, particularly for the immature brain where cerebral autoregulation may be weak or even absent. ${ }^{3}$ The use of inotropic medications for circulatory management and treatment of hypotension in preterm infants is however highly inconsistent, with one study reporting tenfold variability in the use of inotropes between 11 hospitals. ${ }^{4}$ This suggests that inotropic treatment is associated more with the clinician's preference rather than with evidence of effectiveness, and/or the patient's condition. While dopamine is the inotrope most commonly used to treat neonatal hypotension, dobutamine is the most popular second-line therapy, either as monotherapy or together with dopamine. ${ }^{4,5}$ Dobutamine is a synthetic catecholamine that predominantly acts on beta $(\beta)$ adrenoceptors in the myocardium. It has been proposed that dobutamine may have an advantage over dopamine for neonates because of its greater ability to increase cardiac output, and therefore maybe the drug of choice in neonates with myocardial dysfunction. ${ }^{6}$
While the inotropic medications are obviously known to act primarily on the cardiovascular system, very little is known about their effects on the preterm brain. We have previously reported on the potential neuroprotective effect of dopamine in fetal sheep exposed to severe hypoxia causing cerebral injury. ${ }^{7}$ Comparisons between dobutamine-treated and dopamine-treated hypotensive neonates report no difference in short-term outcomes or infant mortality, but the impact on long-term neurodevelopmental outcome is still uncertain. ${ }^{8}$ Indeed, there is little information on the effect of dobutamine on cerebral perfusion in the human preterm brain. In term and preterm normotensive piglets, some studies show that cerebral blood flow is not significantly changed by either dobutamine or dopamine. ${ }^{9}$ Another study describes a maturational effect with dobutamine increasing cerebral blood flow more than dopamine in term piglets, whereas the reverse is true for preterm piglets. ${ }^{10}$

In terms of a direct effect on the brain, in vitro evidence suggests that dobutamine has anti-apoptotic and neuroprotective effects via stimulation of $\beta 1$-adrenoceptors on astrocytes. ${ }^{11}$ Accordingly, dobutamine may also be neuroprotective in the immature brain in the presence of cerebral hypoxia-ischemia. We hypothesized that early and effective treatment of hypotension may avoid organ hypoperfusion and subsequent hypoxic-ischemic injury in the preterm brain. Therefore, we evaluated the effect of pretreatment with dobutamine on cerebral cell death, inflammation, astrogliosis,

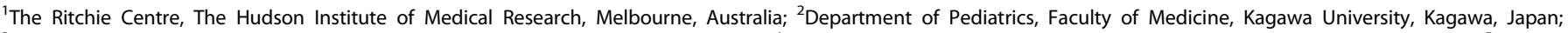

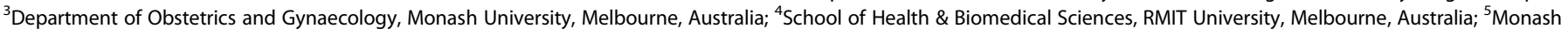
Newborn, Monash Medical Centre, Melbourne, Australia and ${ }^{6}$ Department of Paediatrics, Monash University, Melbourne, Australia Correspondence: Flora Y. Wong (flora.wong@monash.edu)
} 
and oxidative stress induced by hypoxia. We aimed to determine the effect of dobutamine treatment during a severe hypoxic episode, induced by umbilical cord occlusion, in the preterm fetal sheep brain at a stage of development equivalent to $\sim 26-28$ weeks of human gestation. Hypoxia-induced cerebral injury in fetal sheep is a well-characterized and clinically relevant model of the white matter, caudate nucleus, and hippocampal injury often seen in very preterm infants. ${ }^{12-14}$ Diffuse injury in the cerebral white matter in preterm infants is often accompanied by long-term motor impairment, ${ }^{15}$ and injury to the caudate nucleus and hippocampus is considered to predispose preterm infants to cognitive and motor disorders later in life. ${ }^{16,17}$

\section{MATERIALS AND METHODS}

Fetal surgery

All procedures were approved by the Monash University Animal Ethics Committee. At $90 \pm 1$ day of gestation (the term is 147 days), 29 time-mated ewes were prepared for experimentation using aseptic surgical techniques under general anesthesia. Pregnant ewes were anesthetized with intravenous sodium thiopentone $(20 \mathrm{mg} / \mathrm{kg}$; Pentothal), then intubated, and anesthetized using $1-2 \%$ isoflurane in $\mathrm{O}_{2}$. Antibiotics ( $500 \mathrm{mg}$ of ampicillin, $80 \mathrm{mg}$ of gentamicin) were administered during the procedure and daily for 3 days postoperatively. A midline abdominal incision was made, and the fetal head and torso were exposed through a uterotomy. Non-occluding catheters were inserted into one carotid artery and jugular vein in the fetal neck, and a polyvinyl catheter was attached to the fetal skin to measure amniotic pressure. Two electrocardiograph (ECG) electrodes were attached to the fetal chest and an inflatable vascular occluder was placed around the umbilical cord adjacent to the fetal abdomen. Postoperatively, ewes were housed in separate metabolic cages with access to food and water ad libitum, and received analgesia (transdermal fentanyl patch; $75 \mu \mathrm{g} / \mathrm{h}$, Janssen-Cilag) for 3 days. The fetal vascular catheters were maintained patent by continuous infusion of heparinized saline ( 2 units $/ \mathrm{ml}$ at $0.3 \mathrm{ml} / \mathrm{h}$ ).

\section{Monitoring}

Five days post surgery, fetal arterial and amniotic catheters were attached to pressure transducers (ADInstruments) and ECG electrodes were connected to a differential amplifier (FE135 Dual BioAmp, ADInstruments). Fetal mean arterial blood pressure (MAP, corrected for maternal movement and uterine pressure by subtraction of amniotic fluid pressure), and fetal heart rate (HR) derived from the fetal ECG were digitally recorded at $1 \mathrm{kHz}$ (Powerlab 16/30, LabchartPro v7.3.1, ADInstruments) from at least $12 \mathrm{~h}$ before until $72 \mathrm{~h}$ after umbilical cord occlusion (UCO).

\section{Treatment groups}

Experiments were conducted at $95 \pm 1$ day of gestation. Fetuses were randomly assigned to one of the four treatment groups; dobutamine infusion+UCO (Dob+UCO, $n=7$ ), dobutamine infusion+sham occlusion (Dob+sham, $n=6$ ), saline infusion+UCO $(\mathrm{Sal}+\mathrm{UCO}, n=9)$, and saline infusion+sham occlusion (Sal+sham, $n=7)$. Intravenous infusion of dobutamine $(10 \mu \mathrm{g} / \mathrm{kg} / \mathrm{min}$ in 2 units $/ \mathrm{ml}$ heparinized saline), or heparinized saline ( 2 units $/ \mathrm{ml}$ ) was commenced at a rate of $0.5 \mathrm{ml} / \mathrm{h}$ at $2 \mathrm{~h}$ prior to the UCO, and then continued for $74 \mathrm{~h}$ until euthanasia. Fetal weight was assumed to be $1 \mathrm{~kg}$ (based on records from previous work) at the beginning of dobutamine infusion.

Umbilical cord occlusion (UCO)

Two hours after dobutamine or saline infusion commenced, global hypoxia-ischemia was induced by complete UCO. The umbilical occluder was inflated for 25 min with a defined volume of sterile water known to completely occlude blood flow in the umbilical cord. ' Severe hypoxic acidemia in the fetus was confirmed by serial measurement of arterial partial pressure of oxygen $\left(\mathrm{PO}_{2}\right)$ and carbon dioxide $\left(\mathrm{PCO}_{2}\right)$, arterial oxygen saturation, and $\mathrm{pH}$. To avoid fetal death in utero, the UCO was ceased prior to $25 \mathrm{~min}$ if the fetuses developed asystole or became severely hypotensive (MAP $<10 \mathrm{mmHg}$ ).

Fetal arterial blood analysis

Fetal carotid artery blood samples (0.1-0.2 $\mathrm{ml})$ to determine blood $\mathrm{pH}$, gases $\left(\mathrm{PO}_{2}, \mathrm{PCO}_{2}\right.$, and oxygen saturation), and acid-base balance ( $A B L 5000$, Radiometer) were taken daily and at $-2 h,-1 h$, $-15 \mathrm{~min},+5 \mathrm{~min},+20 \mathrm{~min},+2 \mathrm{~h}$, and $+6 \mathrm{~h}$ relative to commencement of UCO.

Tissue collection and preparation

At $72 \mathrm{~h}$ after UCO (i.e., at $98 \pm 1$ day of gestation), the ewe and fetus were euthanized by pentobarbital sodium overdose. The fetus was weighed and the brain was removed, weighed, and divided longitudinally at the midsagittal plane. The right hemisphere was frozen and the left hemisphere was sectioned coronally into $10-\mathrm{mm}$-thick blocks and fixed by immersion in $4 \%$ paraformaldehyde before paraffin embedding. Brain regions for neuropathology analyses (subcortical and periventricular white matter (SCWM and PVWM), hippocampus, and caudate nucleus) were selected topographically using published nomenclature for the sheep brain at the mid-striatal level, corresponding to the sheep brain atlas section number $1000 .{ }^{18}$ In total $10 \mu \mathrm{m}$ thick sections were stained with hematoxylin and eosin to assess gross morphology.

\section{Immunohistochemistry}

Microglia were identified using 1:500 dilution of rabbit antiionized-calcium-binding adaptor molecule (IBA-1: 019-19741, Wako Laboratory Chemicals, Japan). Astrocytes were identified using glial fibrillary acid protein (GFAP) antibody (1:500, Z0334, Dako, Australia). Neurons were identified using anti-NeuN (neuronal nuclei) antibody clone A60 (1:500, MAB377, Merck, Australia). Oxidative damage to DNA and RNA was detected using anti-8-hydroxydeoxyguanosine (1:1000 dilution, 8-OHdG, clone N45.1, JalCA, Japan) antibodies.

All sections were deparaffinized and pretreated with citrate buffer ( $\mathrm{pH}$ 6.0) for $7 \mathrm{~min}$ in the microwave oven to augment antibody-antigen binding. Endogenous peroxidase activity was blocked by incubation with $3 \%$ hydrogen peroxide. Non-specific binding was blocked with $10 \%$ normal goat serum in $0.3 \%$ Triton $\mathrm{X}-100$ in PBS. Sections were subsequently incubated overnight with the primary antibodies at $4{ }^{\circ} \mathrm{C}$. IBA- 1 and GFAP sections were incubated in biotinylated goat anti-rabbit lgG antibody (1:200, BA1000, Vector Laboratories), and anti-NeuN and 8-OHdG sections were incubated in biotinylated goat anti-mouse IgG antibody (1:200, BA-9200, Vector Laboratories) for $60 \mathrm{~min}$. Sections were then reacted using avidin-biotin peroxidase complex kit or streptavidin (Vector Laboratories). The reactive product was visualized using 3,3'-diaminobenzidine (8980681, MP Biomedicals) according to the manufacturer's instructions.

TUNEL assays

Single labeling (Colorimetric) developing cell death with DNA fragmentation was identified using the DeadEnd ${ }^{\mathrm{TM}}$ Colorimetric TUNEL (terminal deoxynucleotidyl transferase dUTP nick-end labeling) system on paraffin sections according to the manufacturer's instructions (G7130, Promega, Australia).

Double-labeling immunofluorescence (Fluorometric) was used to identify cell death with DNA fragmentation on sections from three Sal+UCO fetuses and three Dob+UCO fetuses. TUNEL was performed first using the DeadEnd ${ }^{\mathrm{TM}}$ Fluorometric TUNEL System, according to the manufacturer's instructions (G3250, Promega, Australia), followed by incubation in the GFAP (1:500, Z0334, Dako, Australia) antibody to identify dying astrocytes, and visualized 


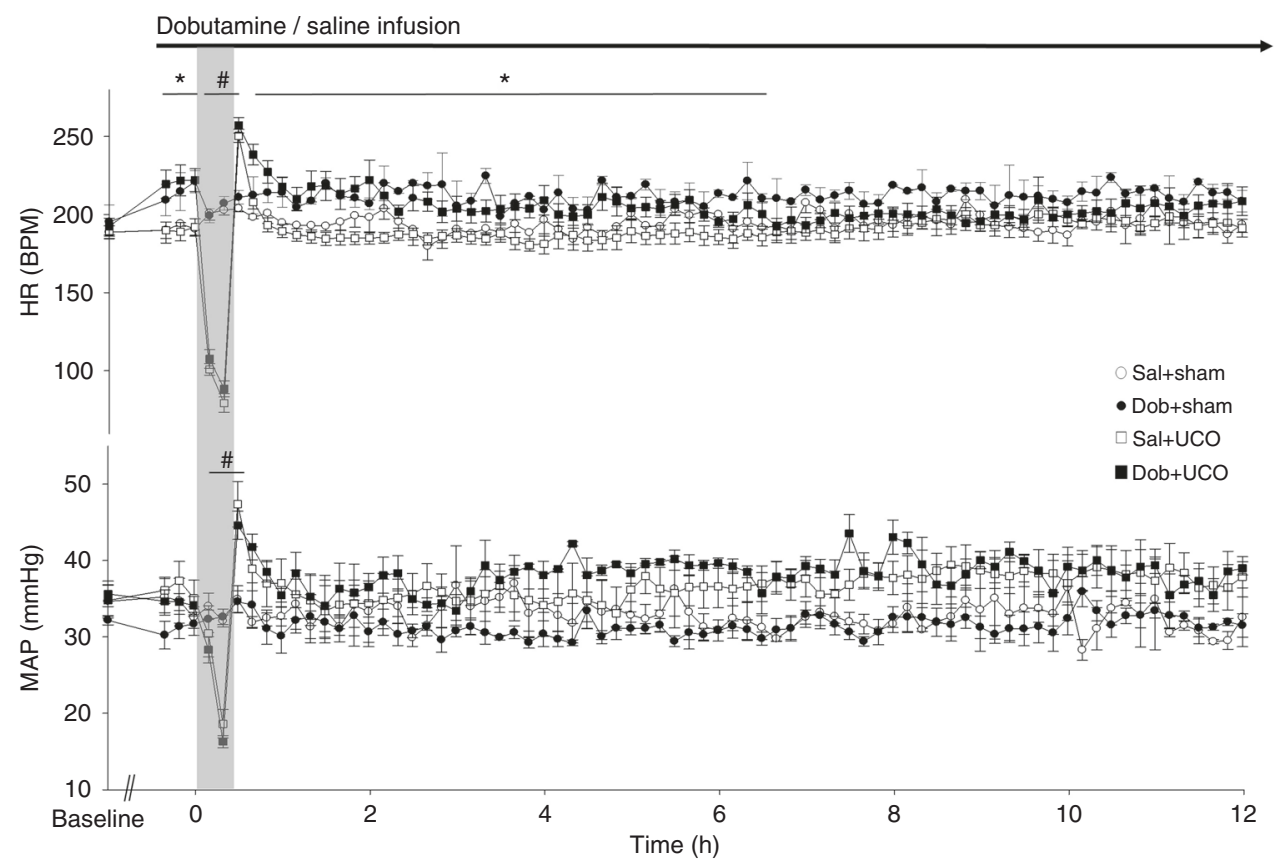

Fig. 1 Heart rate (HR) and mean arterial pressure (MAP) of Sal+sham (O), Dob+sham (O) Sal+UCO ( $\square$ ), and Dob+UCO ( $\square$ ) fetuses, prior to saline or dobutamine infusion (baseline) and before, during, and after umbilical cord occlusion (UCO). Data are mean \pm SEM plotted at 10-min intervals. The gray bar indicates the period of UCO, with time zero at the onset of UCO. *indicates HR of Dob+UCO fetuses significantly greater than Sal+UCO fetuses during the infusion preceding UCO and for $6.5 \mathrm{~h}$ after UCO; \#indicates the difference in HR and MAP between Sal+UCO vs Sal+sham and Dob+UCO vs Dob+sham during UCO and reperfusion

with appropriate Alexa Fluor 594 (1:200 dilution) for $60 \mathrm{~min}$. Double-labeled fluorescent images were visualized using Nikon C1 Digital Eclipse Modular Confocal Microscope System (Nikon Instruments Inc., Japan) and photographs were taken at $\times 200$ magnifications.

Image analysis

Sections were digitally scanned (Image Scope, Aperio Technologies Inc.) and analyzed at $\times 400$ magnification in the SCWM, PVWM, hippocampus, and caudate nucleus by an observer blinded to the experimental groups. IBA-1, GFAP, NeuN, TUNEL, and 8-OHdG immunoreactive cells were manually counted in six nonoverlapping fields $\left(200 \times 200 \mu \mathrm{m}^{2}\right)$ per brain region, on two sections $100 \mu \mathrm{m}$ apart and the results were expressed as the number of cells $/ \mathrm{mm}^{2}$. In addition to the total number of microglia, the number of activated microglia were identified as IBA-1-positive cells with an "ameboid" morphology, ${ }^{19,20}$ usually with a large cell body of round or irregular shape, with short thick branching processes or devoid of branching processes, and expressed as the number of cells $/ \mathrm{mm}^{2}{ }^{19}$ The cross-sectional area occupied by ameboid (activated) microglia was measured in the SCWM and PVWM, and expressed as a percentage of the total combined area of PVWM and SCWM. The percentage of TUNEL-positive cell bodies co-localized for GFAP expression was manually assessed in four non-overlapping fields $\left(200 \times 200 \mu^{2}\right)$ per brain region.

Statistical analysis

Data are presented as mean \pm standard error. Baseline values of fetal MAP and HR were averaged for the 10-min period immediately prior to starting the dobutamine or saline infusion. For the first $180 \mathrm{~min}$ of the infusions (i.e., from $-2 \mathrm{~h}$ to $+1 \mathrm{~h}$ after start of the UCO), fetal MAP and HR were averaged over consecutive 1-min epochs, and thereafter in 10-min epochs until $12 \mathrm{~h}$ after occlusion. Cardiovascular and arterial blood gas data were compared between groups using two-way ANOVA with repeated measures (Sigmastat, version 3.0) and Holm-Sidak post hoc testing. Two-way ANOVA with Tukey post hoc testing was used to compare the immunohistochemical data between groups, with UCO and dobutamine as factors. One-way ANOVA with Tukey post hoc testing was used to compare the immunohistochemical data with our published data on the effect of dopamine treatment with UCO (Dopa+UCO) using the same experimental paradigm. ${ }^{7}$ Statistical significance was accepted when $p<0.05$.

\section{RESULTS}

Brain and body weights

There was no difference in brain weight between groups (Sal +sham, $21.8 \pm 0.4 \mathrm{~g}$; Dob+sham, $21.8 \pm 0.6 \mathrm{~g}$; Sal+UCO, $23.1 \pm 0.9$ $\mathrm{g}$; and Dob+UCO, $20.7 \pm 0.7 \mathrm{~g}$ ). The body weight of Dob+UCO $(1208.6 \pm 94.9 \mathrm{~g})$ was significantly greater than Sal+UCO (987.9 \pm $32.4 \mathrm{~g}$ ) fetuses, likely due to one Dob+UCO fetus being particularly heavy $(1650 \mathrm{~g})$. There was no difference in body weight between Sal+sham $(976.7 \pm 40.6 \mathrm{~g})$ and Dob+sham $(1165.5 \pm 61.2 \mathrm{~g})$ groups.

Cardiovascular results

All fetuses survived the UCO and the 72-h post-UCO period before euthanasia.

$H R$. Baseline HR in Dob+UCO fetuses $(192.3 \pm 5.3 \mathrm{bpm})$ and Sal + UCO fetuses (188.7 $\pm 4.1 \mathrm{bpm}$ ) was not different (Fig. 1). In Dob + UCO fetuses, HR increased significantly to $229.7 \pm 7.7 \mathrm{bpm}$ with dobutamine infusion. During UCO, bradycardia developed to a similar extent in both groups (at 25 min of UCO: Sal+UCO, 71.7 \pm $5.3 \mathrm{bpm}$; Dob+UCO, $79.8 \pm 4.6 \mathrm{bpm}$ ). Following UCO, the Dob + UCO fetuses had an elevated HR for $6.5 \mathrm{~h}$ compared with Sal +UCO group.

MAP. Baseline MAP was not different between Sal+UCO $(35.5 \pm$ $2.3 \mathrm{mmHg})$ and Dob+UCO $(35.6 \pm 1.7 \mathrm{mmHg}$ ) fetuses (Fig. 1). With dobutamine infusion, the MAP did not change significantly compared either to the intra-group pre-infusion level, or to the saline-treated fetuses. During UCO, three of nine Sal+UCO fetuses 


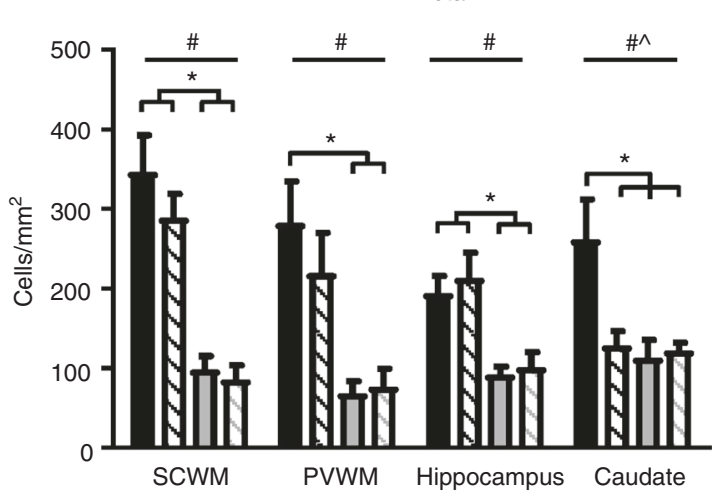

C

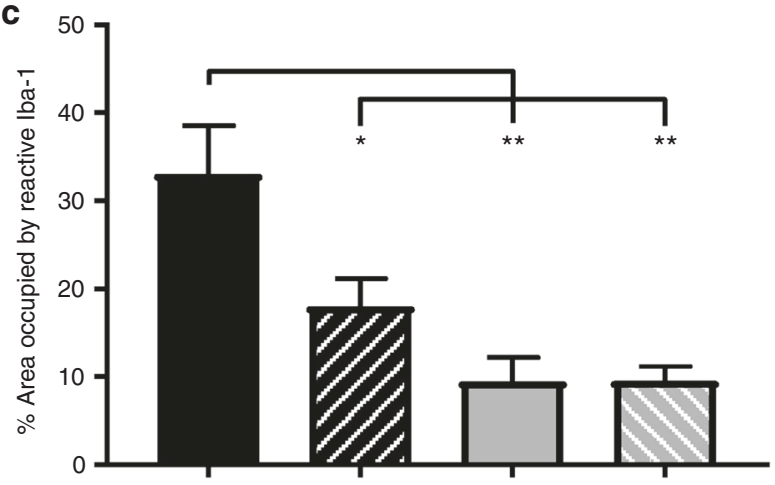

b
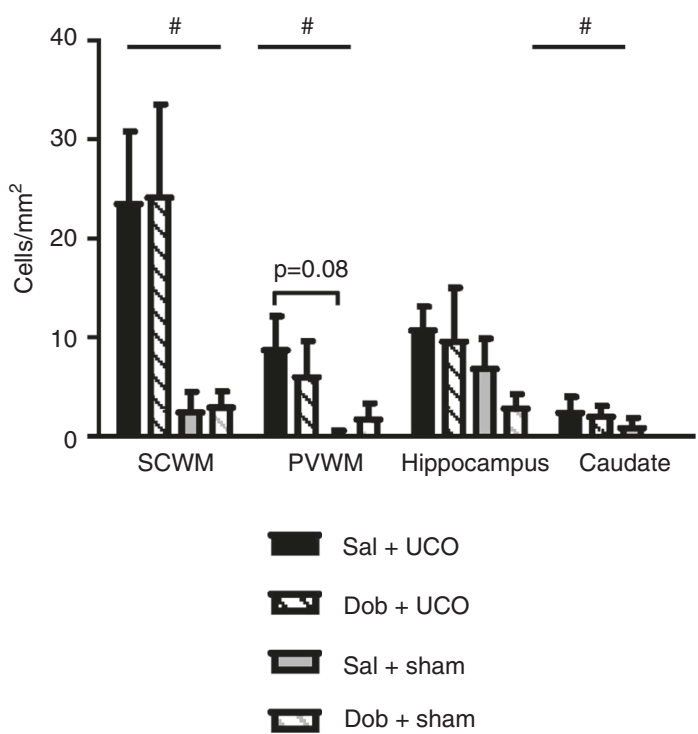

Fig. 2 a Total microglia density and $\mathbf{b}$ activated microglial density in the subcortical white matter (SCWM), periventricular white matter (PVWM), hippocampus, and caudate nucleus, and c percentage area occupied by activated microglia in the combined area of PVWM and SCWM, in the Sal+UCO (black bars), Dob+UCO (black hatched bars), Sal+sham (gray bars), and Dob+sham (gray hatched bars) fetuses. \# $p<$ 0.05 for the effect of UCO, $\wedge p<0.05$ for the effect of dobutamine, ${ }^{*} p<0.05$, ${ }^{* *} p<0.01$ for post hoc analyses, and data are mean \pm SEM

experienced severe hypotension (MAP $<10 \mathrm{mmHg}$ ) at 17,19 , and $21 \mathrm{~min}$, at which point the cuff was deflated and the UCO ended. In comparison, none of the Dob+UCO fetuses developed severe hypotension, and all seven completed 25 min of UCO. For those fetuses that completed $25 \mathrm{~min}$ of UCO, MAP fell to $16.2 \pm 1.5$ $\mathrm{mmHg}$ in the Sal+UCO groups and to $16.1 \pm 1.6 \mathrm{mmHg}$ in Dob + UCO group at $25 \mathrm{~min}$. Post UCO, there were no differences in MAP between the two groups.

\section{Blood gases}

Infusion of dobutamine did not change fetal blood gas parameters in UCO or sham animals. UCO induced severe hypoxia, hypercapnia, acidemia, hypoglycemia, and elevated lactate in Sal + UCO and Dob+UCO fetuses, but the acid-base status of all fetuses recovered quickly after completion of UCO (Supplementary Table S1 (online)). The Dob+sham and Dob+UCO fetuses had higher arterial $\mathrm{PO}_{2}$ than the Sal+sham group at 24 and $72 \mathrm{~h}$ after the UCO, respectively. One Dob+sham fetus had low hemoglobin at $48 \mathrm{~h}(6.3 \mathrm{~g} / \mathrm{dL})$ and $72 \mathrm{~h}(5.9 \mathrm{~g} / \mathrm{dL})$ after sham UCO, but with no HR or blood pressure changes suggestive of hypovolemia (Supplementary Table S1 (online)).

\section{Neuropathology}

Microglia (total). UCO significantly increased the total microglial cell density in all brain regions assessed (Figs. 2a and 3; $p<0.05$ ). Post hoc analysis showed that the microglial density was higher in the Sal+UCO group compared with both sham groups in all brain regions assessed. In the SCWM and hippocampus, microglia density was significantly increased in both UCO groups compared with both sham groups, but in the PVWM, microglia density in the Dob+UCO group was not significantly different compared with the other three groups. In the caudate, the microglial density in the Dob+UCO group was reduced and significantly lower compared with the Sal+UCO animals $(p<0.05)$.

Activated microglia. UCO resulted in a significant increase $(p<$ 0.05 ) in the activated microglial cell density in all regions except the hippocampus (Fig. 2b). The percentage of WM area occupied by aggregations of ameboid (activated) microglia, a marker of diffuse injury, was significantly higher in the Sal+UCO group compared with the Dob+UCO $(p<0.05)$, Sal+sham $(p<0.01)$, and Dob+sham $(p<0.01)$ groups (Figs. $2 c$ and 3$)$. The parenchyma surrounding the areas of dense reactive microglia infiltration also appeared vacuolated in brains from the Sal+UCO fetuses.

Cell death. UCO significantly increased cell death indicated by TUNEL assay in all brain regions assessed $(p<0.05)$. Post hoc analyses showed that cell death was increased in all regions in the Sal+UCO brains in comparison to the sham groups (Figs. 3 and 4a). In the PVWM and caudate nucleus, cell death was also significantly increased in the Dob+UCO brains compared with both sham groups (Fig. 4a).

Neurons. UCO or dobutamine had no effect on the density of NeuN immunoreactive cells in all brain regions examined, in any of the groups (Fig. 4b).

Astrocytes (GFAP). In the hippocampus, dobutamine increased GFAP immunoreactive astrocyte cell density $(p<0.05)$, but post hoc analyses did not identify significant differences between the groups. In the caudate, UCO increased astrocyte cell density $(p<$ $0.05)$, and post hoc analyses showed that astrocyte density of Sal + UCO animals was significantly higher than all other groups $(p<$ 0.05 ) (Figs. 3 and 4c). 

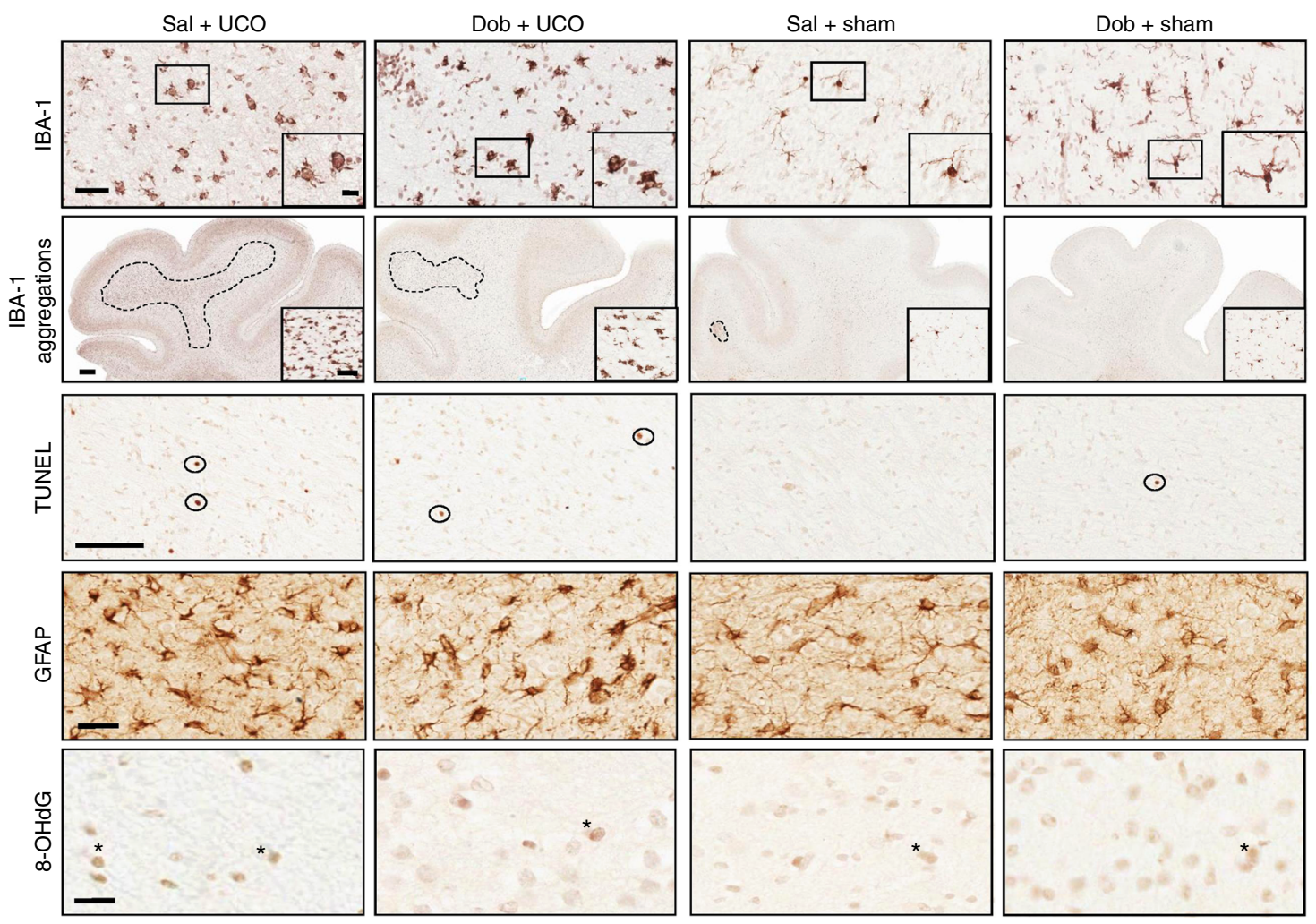

Fig. 3 Images showing immunohistochemistry of IBA-1 (subcortical white matter), areas of IBA-1-positive cell aggregations (in combined periventricular and subcortical white matter), TUNEL (subcortical white matter), GFAP, and 8-OHdG (both in caudate nucleus), in the Sal+UCO, Dob+UCO, Sal+sham, and Dob+sham groups. In the IBA-1 panel, the small squares and their enlarged insets (right lower corners) indicate ameboid-shaped (activated) microglia in the UCO groups, and ramified (resting) microglia in the sham groups. For IBA-1 aggregations, dotted lines indicate areas in the white matter occupied by activated microglia (enlarged insets at right lower corners). In the TUNEL panel, the circles indicate TUNEL-positive cells. In the 8-OHdG panel, *indicates 8-OHdG immunoreactive cells. Scale bars $=50 \mu \mathrm{m}$ for IBA-1 and 20 um for its inset, 400 um for the IBA-1 aggregations and $50 \mu \mathrm{m}$ for its inset, $100 \mu \mathrm{m}$ for TUNEL, and 25 um for GFAP and 8-OHdG

Double-labeled immunohistochemistry revealed that in the Sal + UCO fetuses, $50-70 \%$ of TUNEL-positive cells in all regions were co-localized with GFAP-positive astrocytes (Fig. 5). Similar results were found for the Dob+UCO fetuses (Fig. 5), except in the caudate nucleus where only $36 \pm 18 \%$ of TUNEL-positive cells were co-localized with GFAP, consistent with the lower astrocyte density in the caudate of the Dob+UCO compared with the Sal + UCO group (Fig. 4c).

Oxidative damage (8-OHdG). In the white matter and the hippocampus, there was no difference in $8-\mathrm{OHdG}$ immunoreactivity between the groups. In the caudate, there was an increase in $8-\mathrm{OHdG}$ immunoreactivity in the UCO groups $(p<0.05)$ (Figs. 3 and $4 d)$.

\section{Comparison with dopamine treatment}

The data obtained for TUNEL and IBA-1 immunostaining were compared with those that we obtained using the same experimental paradigm of UCO in fetal sheep, but with dopamine infusion (Dopa+UCO) instead of dobutamine ${ }^{7}$ (Fig. 6). Compared to the Sal+sham group, cell death was significantly higher in the Sal+UCO group in all brain regions examined, significantly higher in the Dob+UCO group in PVWM and caudate, and higher in the Dopa+UCO group in the caudate only (Fig. 6a). Microglia (total) density (Fig. 6b) was increased in the Sal+UCO group compared with the Sal+sham group in all regions. Dob+UCO group also had increased total microglia density than the Sal+sham group in SCWM and hippocampus. In the caudate, both the Dob+UCO and Dopa+UCO groups had lower total microglia density than the Sal+UCO group. The Dopa+UCO group also showed a trend of lower total microglial density than the Sal+UCO group in SCWM $(p=0.06)$, and was lower than both Sal+UCO and Dob+UCO groups in the hippocampus ( $p=0.07$ and 0.05 , respectively), but just failed to reach statistical significance. Activated microglial density also tended to be higher in the Sal+UCO group in both SCWM and PVWM ( $p=0.05$ for both), and higher in the Dob+UCO group in the SCWM $(p=0.08)$, compared with the Sal+sham group (Fig. 6c). Compared with the Sal+sham group, the Dopa+UCO group did not show higher microglia density (total or activated) in any region.

\section{DISCUSSION}

To our knowledge, this is the first study to evaluate the effects of dobutamine on neuropathy induced by cerebral hypoxia in the immature brain. A clinically relevant model of hypoxia-induced brain injury was used, in which the neuropathological changes are similar to those in preterm human infants. ${ }^{14}$ Uncertainty remains regarding whether and when hypotension or suboptimal cardiac output requires early treatment; so in this study, dobutamine was administered prior to UCO to determine if early treatment in infants at the risk of circulatory compromise affects subsequent cerebral hypoxic injury. Our results show that intravenous dobutamine, begun before the onset of a severe asphyxial insult, reduced the area occupied by activated microglia in cerebral white matter and the total microglial and astrocyte density in the caudate. Dobutamine pretreatment did not exacerbate cerebral hypoxic injury, or induce cell death, microglial infiltration, or astrogliosis injury in the sham group, as measured by the immunohistological markers used in this study. 


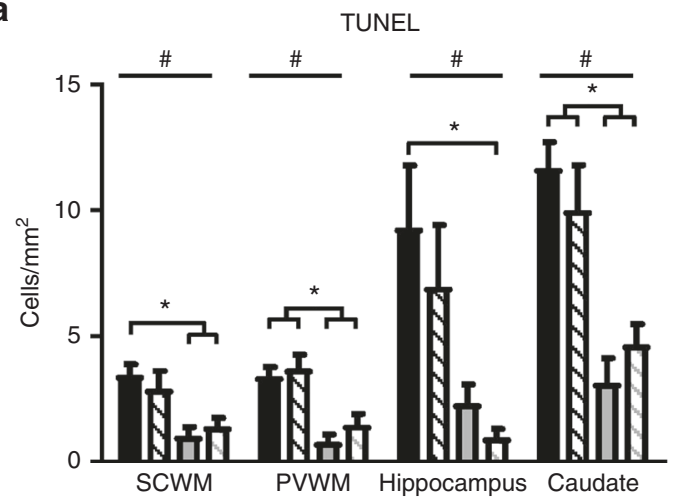

C

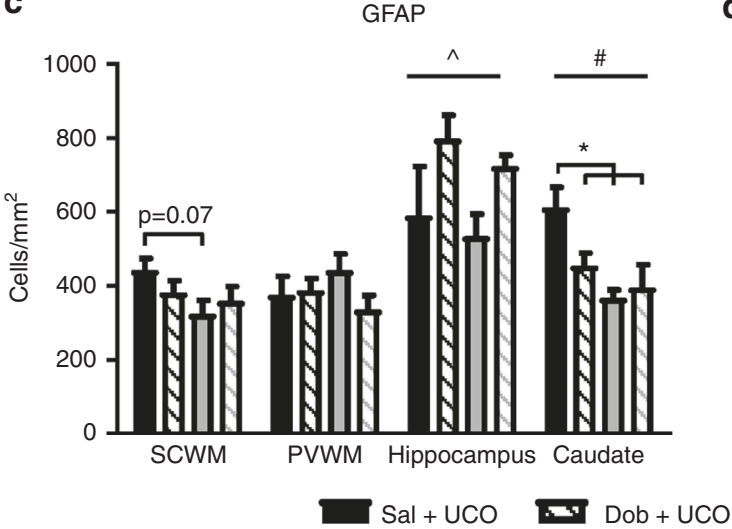

b

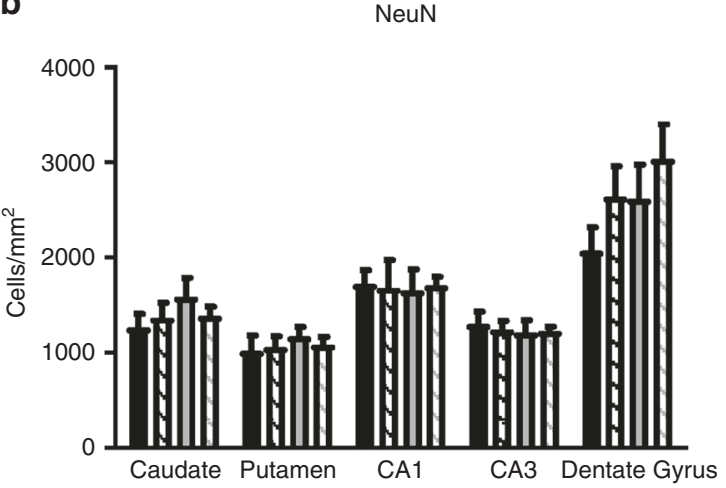

d

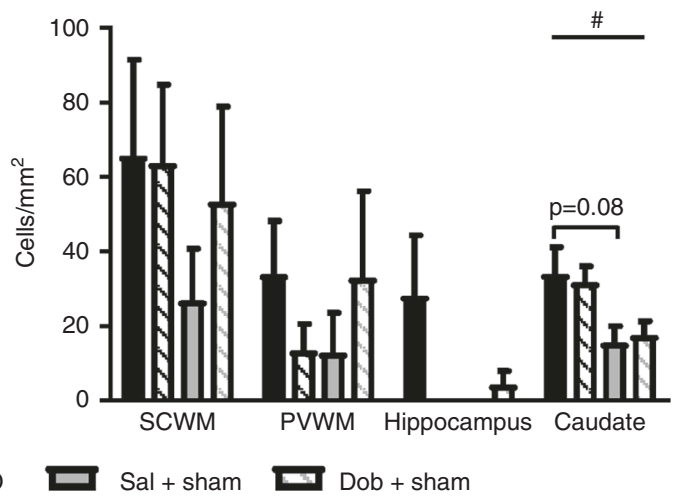

Fig. 4 a Cell death detected by TUNEL assay, b Neu-N immunoreactive cell density, c GFAP immunoreactive astrocyte density, and d 8-OHdG immunoreactive cell density in the SCWM, PVWM, hippocampus, and caudate nucleus, in the Sal+UCO (black bars), Dob+UCO (black hatched bars), Sal+sham (gray bars), and Dob+sham (gray hatched bars) groups at $72 \mathrm{~h}$ after UCO. ${ }^{\#} p<0.05$ for the effect of UCO, $\wedge p<0.05$ for the effect of dobutamine, ${ }^{*} p<0.05$ for post hoc analyses, and data are means \pm SEM
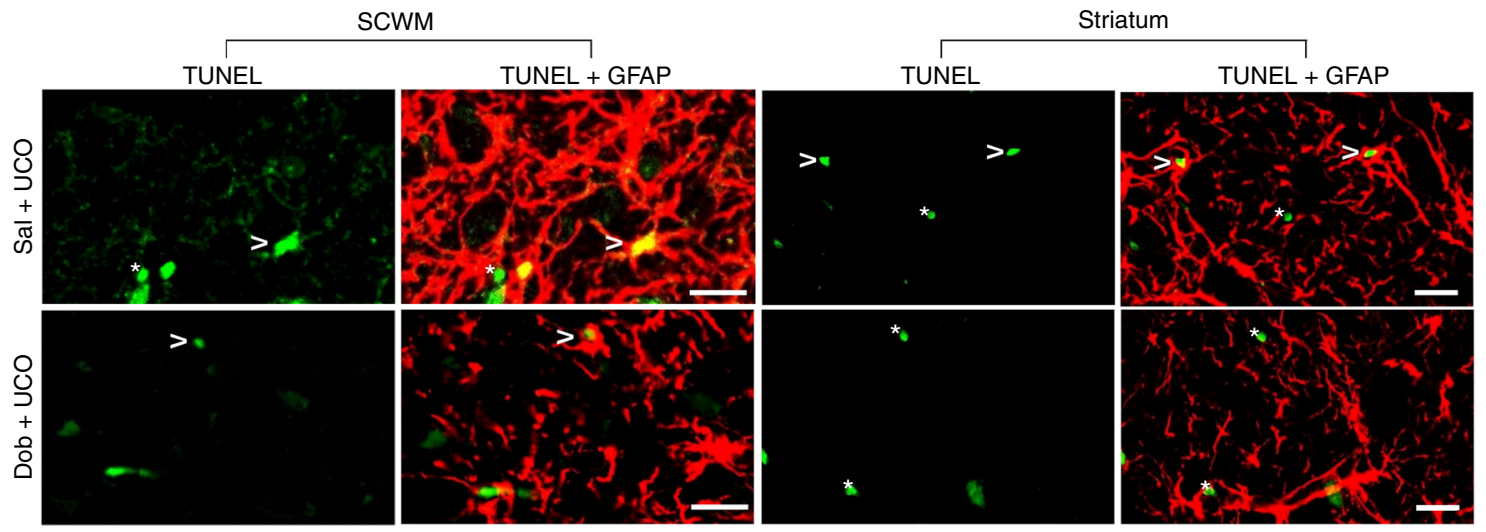

Fig. 5 Confocal images showing immunohistochemistry of TUNEL (green) and double immunofluorescence (merge) with GFAP (red), in SCWM and striatum of the UCO groups. ${ }^{*}$ indicates TUNEL-positive cell bodies not co-localized for GFAP, and $>$ indicates cell bodies which are co-localized for GFAP and TUNEL. Scale bars $=20 \mu \mathrm{m}$

There are several mechanisms by which dobutamine may affect cerebral hypoxic injury. Studies in the human newborn and piglets show that dobutamine infusion at $10 \mu \mathrm{g} / \mathrm{kg} / \mathrm{min}$ increases cerebral blood flow and cerebral oxygenation. ${ }^{21,22}$ The cerebral hemodynamic effects are likely due to the increase in systemic blood pressure and/or cardiac output, leading to higher cerebral blood flow, as dobutamine does not have any direct vasomotor effect on brain microvessels. ${ }^{23}$ Cerebral hemodynamic measurements by near-infra-red spectroscopy ${ }^{24}$ may help to further define the cerebrovascular effects of dobutamine. Alternatively, dobutamine increases systemic oxygen consumption and oxygen extraction in newborn lambs. ${ }^{25}$ If similar effects occurred in the preterm brain, the increased cerebral oxygen extraction may lead to, and/or exacerbate cerebral hypoxia. However, dobutamine-treated animals in both the UCO and sham groups did not show increased cell death, suggesting that dobutamine did not contribute to hypoxic brain injury.

Dobutamine reduces neuronal cell death and inflammation in newborn murine hippocampal slice cultures subjected to hypoxia-ischemia. ${ }^{26} \beta 1$-adrenergic stimulation by dobutamine also protects against glutamate-induced excitotoxicity, a key mediator of neuronal loss consequent to neonatal 
TUNEL

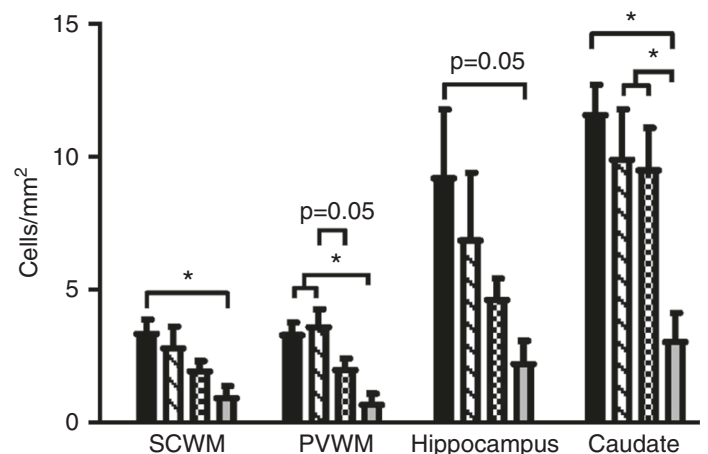

C

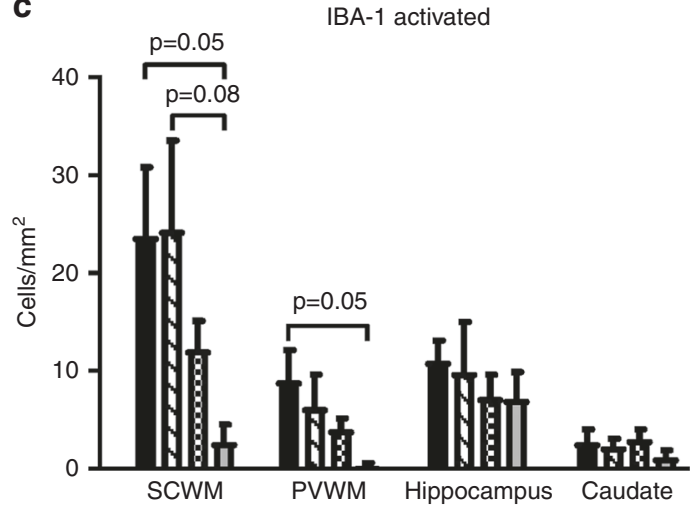

b

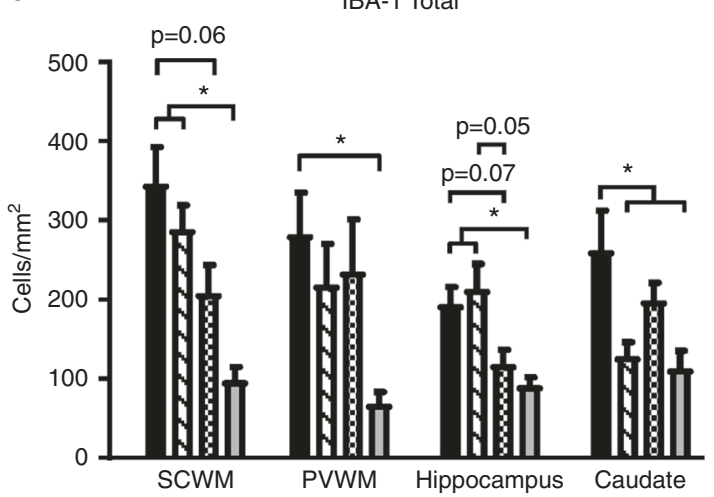

Fig. 6 Comparison with data from fetal sheep obtained using the same experimental paradigm, but with dopamine instead of dobutamine infusion before UCO (Dopa+UCO). ${ }^{7}$ a Cell death was detected by TUNEL assay, b total microglia density, and $\mathbf{c}$ activated microglial density in the SCWM, PVWM, hippocampus, and caudate nucleus, in the Sal+UCO (black bars), Dob+UCO (black hatched bars), Dopa+UCO (checked bars), and Sal+sham (gray bars) fetuses, at $72 \mathrm{~h}$ after UCO. ${ }^{*} p<0.05$, data are mean \pm SEM

hypoxic-ischemic insults, ${ }^{27}$ in mixed neuronal-glial cultures. ${ }^{11}$ However, it is unclear if intravenous dobutamine crosses the immature blood-brain barrier and directly acts on adrenoreceptors in the brain. Further studies are required to assess the cerebral concentration of dobutamine during intravenous dobutamine infusion. While we detected reduced microglia, there was no difference in neuron cell density and cell death with dobutamine infusion.

Astrocytes are the main cell-type-expressing $\beta$-adrenoceptors in the brain and $\beta$-adrenoceptor activation drives astrogliosis. ${ }^{28}$ While both $\beta 1$ - and $\beta 2$-adrenoceptor stimulations in hippocampal cell cultures induce astrocyte activation associated with neuroprotection against glutamate excitotoxicity, in vivo neuroprotection was mediated by $\beta 2$-stimulation. ${ }^{11}$ In our study of the immature sheep brain, dobutamine increased astrocyte density in the hippocampus, but the increased astrocyte density in the caudate nucleus following UCO was significantly reduced in the Dob+UCO animals. These findings suggest a regionspecific effect of the astrocyte proliferation in response to dobutamine.

$\beta$-adrenoceptor stimulation increases antioxidant glutathione in cultured neurons, rendering protection against oxidative stress. ${ }^{29}$ However, using 8-OHdG as a marker of oxidative stress, we did not observe any effects of dobutamine in either the sham or UCO groups.

Using the same experimental paradigm, we previously reported that dopamine treatment reduced cell death and microglia in the fetal sheep brain after severe cerebral hypoxia. ${ }^{7}$ In comparison with dopamine treatment, dobutamine was less effective in reducing cell death in the PVWM and microglial density in the SCWM and hippocampus. Both dopamine and dobutamine reduced microglial density in the caudate nucleus. In preterm piglets, blood flow to the heart and brain is augmented by 10 and $15 \mu \mathrm{g} / \mathrm{kg} / \mathrm{min}$ dopamine infusion but not by dobutamine at these doses. ${ }^{10}$ The different effects of dopamine and dobutamine may be due to their different receptor targets; dopamine stimulates both $\alpha$ - and $\beta$-adrenoceptors, while dobutamine acts primarily on $\beta$-adrenoceptors. $a$-Adrenoceptor sensitivity develops before $\beta$ adrenoceptors, $^{30}$ and therefore may facilitate the action of dopamine in the preterm circulation. In addition, the expression of $\beta 1$-adrenoceptors in the preterm piglet heart is less than that in the term heart, ${ }^{31}$ and this would limit the preterm responses to $\beta$ adrenergic agonists such as dobutamine. There is also a possibility that dopamine and dobutamine administered to the fetus may cross over the placenta into the maternal circulation. We have shown that dopamine administered to the fetal sheep at $10 \mu \mathrm{g} /$ $\mathrm{kg} / \mathrm{min}$ increased the fetal plasma dopamine up to 300 -fold, similar to the increase in plasma dopamine levels in human newborns administered with $5-10 \mu \mathrm{g} / \mathrm{kg} / \mathrm{min}$ of dopamine. ${ }^{32}$ This finding suggested that dopamine did not cross over to the maternal circulation in significant amounts. Dobutamine has a higher molecular weight than dopamine (301.38 Da vs 153.18 Da) and is therefore not likely to cross over to the maternal circulation, as in the case of dopamine. However, we did not assess the fetal plasma level of dobutamine to confirm this.

In this study, dobutamine at the mid-clinical dose of $10 \mu \mathrm{g} / \mathrm{kg} /$ min increased HR, but not MAP. The negligible MAP response may be due to the umbilical-placental circulation, which at 0.6 gestation contains over half of the fetoplacental blood volume. ${ }^{33}$ Thus, the lack of MAP response, as we and others have reported using inotropic agents in fetal sheep, ${ }^{7,30}$ may be due to the displacement of fetal blood into the low-resistance vascular bed of 
the umbilical-placental circulation. Continuous hemodynamic measurements in the fetal sheep by insertion of transonic flow probes, $^{34}$ e.g., around the aorta to measure left ventricular output, may help to further define the cardiovascular effects of dobutamine infusion. Notably, studies in preterm piglets infused with a similar dose of dobutamine also reported increased HR without changes to MAP or cardiac output., ${ }^{9,10}$ In preterm human infants, dobutamine increases HR and cardiac output in a dosedependent manner but with inconsistent effects on arterial blood pressure. $^{35,36}$

Heart rate was normalized in fetal sheep infused with dobutamine at $6.5 \mathrm{~h}$ after the UCO, similar to our previous report in the fetal sheep infused with dopamine. ${ }^{7}$ In preterm human infants, there is the possibility of desensitization of the cardiovascular system to catecholamines, as shown by the development of inotrope-resistant hypotension, ${ }^{37}$ but it remains unclear if this occurs because of tissue hypoxia, or downregulation of adrenoceptors due to their prolonged exposure to adrenoceptor agonists. ${ }^{38}$ Interestingly, Drury et al. reported that following UCO in fetal lambs, dopamine administration delayed, but did not prevent terminal hypotension and death by $\sim 6 \mathrm{~h}^{39}$ This finding, together with our results, suggests that the preterm fetal lamb heart may become desensitized to adrenergic agonists after $6 \mathrm{~h}$. The desensitization with prolonged use of catecholamines, whether the medication started before or after the circulatory compromise, may underlie inotrope-resistant hypotension. Hydrocortisone administration is an effective adjunctive treatment of neonatal hypotension and reduces the dosage of adrenergic agonists required. ${ }^{37}$ One of the major genomic effects of glucocorticoids is upregulation of adrenoceptors and their second messenger systems, thus increasing the sensitivity of the preterm cardiovascular system to adrenergic agonists and stabilizing the circulatory status.

Our study addressed the important clinical question of whether dobutamine pretreatment alters the vulnerability of the preterm brain to injury arising from cardiovascular instability and cerebral hypoxia. Notably, the practice of "permissive hypotension" has been raised in recent years, which is at least partly based on the concern that use of inotropic medication to increase blood pressure may have adverse effects. ${ }^{40}$ Our study is therefore important in addressing this issue of potential benefit and adverse effect of early dobutamine treatment on the brain. Clinical trials of permissive hypotension had significant difficulties with patient recruitment, including consent issues and physician willingness to enroll. ${ }^{40}$ Currently, there remains diversity in the timing of administration of inotropic medications among neonatologists. Dobutamine is often used clinically as a rescue therapy in preterm infants who suffer cardiovascular instability and hypoxia-ischemia. A limitation of our study is that it does not address whether dobutamine has similar effects when treatment is commenced at different time points before or after the cerebral hypoxic insult. Importantly, our results show that dobutamine mitigates hypoxiainduced microglial infiltration in the white matter, and reduces microglia density and astrogliosis in the caudate nucleus. Dobutamine itself does not induce or exacerbate cell death, microglial infiltration, or astrogliosis in the preterm brain. Therefore, early administration of dobutamine in preterm infants at the risk of circulatory compromise appears safe without compounding subsequent hypoxic-ischemic brain injury.

\section{ACKNOWLEDGEMENTS}

We thank Ms Meghan Boomgardt for technical assistance with the immunohistochemistry. This research was supported by funding from National Health and Medical Research Council (NHMRC project Grant APP 1025626) of Australia and the Victorian Government's Operational Infrastructure Support Program. F.Y.W. is supported by the NHMRC/Cerebral Palsy Alliance Career Development Fellowship (APP 1084254).

\section{ADDITIONAL INFORMATION}

The online version of this article (https://doi.org/10.1038/s41390-018-0045-5) contains supplementary material, which is available to authorized users.

Competing interests: The authors declare no competing interests.

Publisher's note: Springer Nature remains neutral with regard to jurisdictional claims in published maps and institutional affiliations.

\section{REFERENCES}

1. Omizzolo, C. et al. Neonatal brain abnormalities and memory and learning outcomes at 7 years in children born very preterm. Memory 22, 605-615 (2014).

2. Back, S. A., Riddle, A. \& McClure, M. M. Maturation-dependent vulnerability of perinatal white matter in premature birth. Stroke 38, 724-730 (2007).

3. O'Leary, H. et al. Elevated cerebral pressure passivity is associated with prematurity-related intracranial hemorrhage. Pediatrics 124, 302-309 (2009).

4. Lasky, T., Greenspan, J., Ernst, F. R. \& Gonzalez, L. Dopamine and dobutamine use in preterm or low birth weight neonates in the premier 2008 database. Clin. Ther. 33, 2082-2088 (2011).

5. Rios, D. R., Moffett, B. S. \& Kaiser, J. R. Trends in pharmacotherapy for neonatal hypotension. J. Pediatr. 165, 697-701 (2014).

6. Noori, S. \& Seri, I. Neonatal blood pressure support: the use of inotropes, lusitropes, and other vasopressor agents. Clin. Perinatol. 39, 221-238 (2012).

7. Brew, N. et al. Dopamine treatment during acute hypoxia is neuroprotective in the developing sheep brain. Neuroscience 316, 82-93 (2016).

8. Subhedar N. V., Shaw N. J. Dopamine versus dobutamine for hypotensive preterm infants. Cochrane Database Syst. Rev. CD001242 (2003).

9. Eiby, Y. A. et al. Inotropes do not increase cardiac output or cerebral blood flow in preterm piglets. Pediatr. Res. 80, 870-879 (2016).

10. Ferrara, J. J. et al. Effects of dopamine and dobutamine on regional blood flow distribution in the neonatal piglet. Ann. Surg. 221, 531-540 (1995). discussion 540-532.

11. Junker, V. et al. Stimulation of beta-adrenoceptors activates astrocytes and provides neuroprotection. Eur. J. Pharmacol. 446, 25-36 (2002).

12. Drury, P. P. et al. Partial neural protection with prophylactic low-dose melatonin after asphyxia in preterm fetal sheep. J. Cereb. Blood Flow. Metab. 34, 126-135 (2014).

13. Jellema, R. K. et al. Cerebral inflammation and mobilization of the peripheral immune system following global hypoxia-ischemia in preterm sheep. J. Neuroinflamm. 10, 13 (2013).

14. Mallard, C., Welin, A. K., Peebles, D., Hagberg, H. \& Kjellmer, I. White matter injury following systemic endotoxemia or asphyxia in the fetal sheep. Neurochem. Res. 28, 215-223 (2003).

15. Spittle, A. J. et al. Neonatal white matter abnormality predicts childhood motor impairment in very preterm children. Dev. Med. Child Neurol. 53, 1000-1006 (2011).

16. Abernethy, L. J., Cooke, R. W. \& Foulder-Hughes, L. Caudate and hippocampal volumes, intelligence, and motor impairment in 7-year-old children who were born preterm. Pediatr. Res. 55, 884-893 (2004).

17. Beauchamp, M. H. et al. Preterm infant hippocampal volumes correlate with later working memory deficits. Brain 131, 2986-2994 (2008).

18. Johnson J. The Sheep Brain Atlas (online). (Mishigan State University Brain Biodiversity Bank, 2008).Available: http://www.msu.edu/brains/sheep/index.html

19. Hutton, L. C., Castillo-Melendez, M. \& Walker, D. W. Uteroplacental inflammation results in blood brain barrier breakdown, increased activated caspase 3 and lipid peroxidation in the late gestation ovine fetal cerebellum. Dev. Neurosci. 29, 341-354 (2007).

20. Yawno, T. et al. Human amnion epithelial cells protect against white matter brain injury after repeated endotoxin exposure in the preterm ovine fetus. Cell Transplant. 26, 541-553 (2017).

21. Nachar, R. A. et al. Dose-dependent hemodynamic and metabolic effects of vasoactive medications in normotensive, anesthetized neonatal piglets. Pediatr. Res. 70, 473-479 (2011)

22. Robel-Tillig, E., Knupfer, M., Pulzer, F. \& Vogtmann, C. Cardiovascular impact of dobutamine in neonates with myocardial dysfunction. Early Hum. Dev. 83, 307-312 (2007).

23. Kawamura, S. \& Yasui, N. Effects of dobutamine on brain surface microvessels in rats. Neurol. Med. Chir. 38, 137-141 (1998).

24. Bennet, L., Roelfsema, V., Pathipati, P., Quaedackers, J. S. \& Gunn, A. J. Relationship between evolving epileptiform activity and delayed loss of mitochondrial activity after asphyxia measured by near-infrared spectroscopy in preterm fetal sheep. $J$. Physiol. 572, 141-154 (2006). 
25. Penny, D. J., Sano, T. \& Smolich, J. J. Increased systemic oxygen consumption offsets improved oxygen delivery during dobutamine infusion in newborn lambs. Intensive Care Med. 27, 1518-1525 (2001).

26. Markus, $T$. et al. beta-Adrenoceptor activation depresses brain inflammation and is neuroprotective in lipopolysaccharide-induced sensitization to oxygen-glucose deprivation in organotypic hippocampal slices. J. Neuroinflamm. 7, 94 (2010).

27. Hagberg, H., Peebles, D. \& Mallard, C. Models of white matter injury: comparison of infectious, hypoxic-ischemic, and excitotoxic insults. Ment. Retard. Dev. Disabil. Res. Rev. 8, 30-38 (2002).

28. Hodges-Savola, C., Rogers, S. D., Ghilardi, J. R., Timm, D. R. \& Mantyh, P. W. Betaadrenergic receptors regulate astrogliosis and cell proliferation in the central nervous system in vivo. Glia 17, 52-62 (1996).

29. Madrigal, J. L., Kalinin, S., Richardson, J. C. \& Feinstein, D. L. Neuroprotective actions of noradrenaline: effects on glutathione synthesis and activation of peroxisome proliferator activated receptor delta. J. Neurochem. 103, 2092-2101 (2007).

30. Gleason, C. A., Robinson, R., Harris, A. P., Mayock, D. E. \& Traystman, R. J. Cerebrovascular effects of intravenous dopamine infusions in fetal sheep. J. Appl. Physiol. 92, 717-724 (2002).

31. Kim, M. Y. et al. Expression of adrenoceptor subtypes in preterm piglet heart is different to term heart. PLOS ONE 9, e92167 (2014).
32. Olhager, E. et al. Preterm lambs given intravenous dopamine show increased dopamine in their cerebrospinal fluid. Acta Paediatr. 103, 337-342 (2014).

33. Creasy, R. K., Drost, M., Green, M. V. \& Morris, J. A. Determination of fetal, placental and neonatal blood volumes in the sheep. Circ. Res. 27, 487-494 (1970).

34. Crossley, K. J. et al. Dynamic changes in the direction of blood flow through the ductus arteriosus at birth. J. Physiol. 587, 4695-4704 (2009).

35. Bravo, M. C. et al. Randomized, placebo-controlled trial of dobutamine for low superior vena cava flow in infants. J. Pediatr. 167, 572-578 (2015).

36. Osborn, D., Evans, N. \& Kluckow, M. Randomized trial of dobutamine versus dopamine in preterm infants with low systemic blood flow. J. Pediatr. 140, 183-191 (2002)

37. Noori, S. et al. Hemodynamic changes after low-dosage hydrocortisone administration in vasopressor-treated preterm and term neonates. Pediatrics 118, 1456-1466 (2006)

38. Hausdorff, W. P., Caron, M. G. \& Lefkowitz, R. J. Turning off the signal: desensitization of beta-adrenergic receptor function. FASEB J. 4, 2881-2889 (1990).

39. Drury, P. P. et al. Dopamine infusion for postresuscitation blood pressure support after profound asphyxia in near-term fetal sheep. Exp. Physiol. 98, 699-709 (2013).

40. Dempsey, E. M. Challenges in treating low blood pressure in preterm infants. Children 2, 272-288 (2015) 